\title{
PROYECCIONES Y ALCANCES PSICOTERAPÉUTICOS DE LA DOCTRINA EVAGRIANA DE LA SALUD DEL ALMA. UN ANÁLISIS DESDE EL ESTATUTO EPISTEMOLÓGICO DE LA PSICOTERAPIA
}

\author{
PROJECTIONS AND PSYCHOTHERAPEUTIC SCOPES OF THE \\ EVAGRIAN DOCTRINE OF THE HEALTH OF THE SOUL. AN \\ ANALYSIS FROM THE EPISTEMOLOGICAL STATUTE OF \\ PSYCHOTHERAPY
}

\author{
MARÍA TERESA GARGIULO \\ Conicet - Universidad Nacional de Cuyo \\ SANTIAGO HERNÁN VÁZQUEZ \\ Conicet - Universidad Nacional de Cuyo
}

\section{RESUMEN}

En los últimos años ha crecido el número de estudios abocados a analizar en los escritos de Evagrio Póntico el potencial psicoterapéutico de su doctrina. Ahora bien, cabe preguntarse si dichas investigaciones no representan una extensión epistemológicamente ilegitima de prácticas que originalmente fueron pensadas con una intencionalidad primariamente ético-religiosa. En este trabajo mostraremos que nuestro autor no sólo reconoce en el alma enferma una etiología eminentemente teológica, sino que se preocupa por formular prácticas psicoterapéuticas que atienden a dinámicas propiamente psíquicas. En su doctrina de los ocho logismoi propone una serie de prácticas en orden a curar los procesos de desequilibrio pasional y de distorsión cognitiva a los que dicho estado de enfermedad espiritual da lugar. A nuestro entender la particular comprensión que tiene el monje del Ponto respecto de la psicoterapia que practica representa verdaderas y auténticas 
proyecciones para la compleja discusión, de nuestros días, acerca del estatuto epistemológico de la psicoterapia.

Palabras clave: Psicoterapia; Espiritualidad; Evagrio Póntico; Psicología Positiva; Problema religioso o espiritual.

\section{ABSTRACT}

In recent years, the number of studies devoted to analyzing in Evagrio Póntico's writings the psychotherapeutic potential of his doctrine has increased. Now, it is worth asking whether these investigations do not represent an epistemologically illegitimate extension of practices that were originally thought with a primarily ethical-religious intentionality. In this work we will show that our author not only recognizes in the diseased soul an eminently theological etiology but also worries about formulating psychotherapeutic practices that attend to properly psychic dynamics. In his doctrine of the eight logismoi proposes a series of practices in order to cure the processes of passional imbalance and cognitive distortion to which this state of spiritual illness gives rise. In our opinion, the particular understanding that the monk of Pontus has regarding the psychotherapy he practices represents real and authentic projections for the complex discussion, of our days, about the epistemological status of psychotherapy.

Keywords: Psychotherapy; Spirituality; Evagrio Ponticus; Positive Psychology; Religious or spiritual problem.

\section{INTRODUCCIÓN}

Existen en nuestros días cuantiosos estudios que procuran medir cómo las prácticas espirituales promueven la salud física y mental. Diversas tradiciones o escuelas psicoterapéuticas presentan la religión y la espiritualidad como importantes mecanismos que tienen efectos directos e indirectos sobre la salud humana.

En este marco puede interpretarse la reciente y vasta literatura que ha surgido en nuestros días con el propósito de visualizar las connotaciones psicoterapéuticas de ciertas prácticas antiguas de espiritualidad tales como las del primer monacato cristiano. En las últimas décadas, ha crecido puntualmente el in-

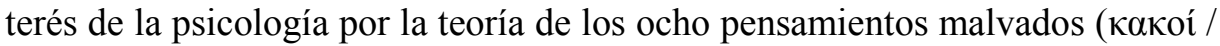
$\lambda$ o $16 \mu$ oí) formulada por Evagrio Póntico (Larchet 2003; 2006; Corrigan 2017; Graiver 2016, 2018; Tsakiridis 2010; Hill 2010; Trader 2012; Bradford 2012; 
2011; Gianfrancesco 2008; Peretó Rivas 2017; Vazquez 2015). De una u otra manera estos estudios destacan el valor terapéutico de esta doctrina evagriana en orden a erradicar lo que el autor reconoce como el sustrato cognitivo-emocional del alma enferma

Ahora bien, frente a este tipo de estudio cabe plantear la siguiente cuestión. ¿La cura del alma que propone Evagrio posee realmente valor psicoterapéutico? Las investigaciones citadas que postulan el potencial psicoterapéutico de su doctrina ¿no están traspasando a un modelo médico, una doctrina que originariamente ha sido formulada bajo un estatuto ético-teológico? ¿Acaso este tipo de lecturas no representan una extensión epistemológicamente ilegítima de las metáforas medicas a las que alude repetidas veces el autor en su obra? ¿No estarían, en este sentido, proyectando de un modo ambiguo y difuso al campo de la psicoterapia una práctica que inicialmente fue pensada en un contexto ético-religioso?

Desde los primeros diálogos platónicos hasta las grandes obras del estoicismo antiguo se sitúa el cuidado de sí o el cuidado del alma en el marco de una reflexión específicamente moral. Ahora, en este tipo de abordaje es habitual, tanto en el ámbito filosófico como en el bíblico y el patrístico, hacer uso de la metáfora médica. Los desarrollos de Evagrio no parecen ser una excepción en este sentido. Sus escritos repetidas veces presentan esta extendida práctica de la filosofía de ilustrar principios morales o espirituales mediante analogías basadas sobre la teoría y el vocabulario de la medicina clásica.

En pocas palabras: ¿cuándo Evagrio dice ocuparse de las dolencias del alma, ello tiene una connotación realmente psicoterapéutica o solo debe entenderse en un sentido metafórico?

La cuestión que aquí planteamos ha sido objeto de estudio de abundante literatura. Entre ellos cabe citar, los estudios de Laín Entralgo (2005, 128), García Ballester (1996, 724), Larchet (2001, 2007), Ramelli (2015), Fernandez (1999) y Dysinger $(2004 ; 2005,104)$. De una u otra manera los citados estudios procuran demostrar que los antiguos griegos y, particularmente, el primer monacato cristiano, al predicar la noción de enfermedad respecto de ciertos estados del alma no está haciendo uso necesariamente de un mero recurso metafórico sino que, más bien, busca expresar algo más vinculante y profundo. El alma humana al padecer un desequilibrio en sus pasiones, y en sus estructuras y dinámicas cognitivas se encuentra real y propiamente enferma. En el presente trabajo procuraremos presentar -en línea con esta misma hipótesis- el valor psicoterapéutico de los escritos de Evagrio, a través de una dilucidación acerca del estatuto epistemológico de la psicoterapia. Ciertamente en los escritos evagrianos 
encontramos un tratamiento profundo y extenso sobre el alma humana; un horizonte teológico y filosófico que da cuenta del lógos del ánthropos. Pero lo que nos interesa puntualmente indagar es si existe en su obra el desarrollo de una particular práctica psicoterapéutica y de ser así, bajo qué estatuto epistemológico la concibe. Para ello atenderemos no sólo a sus tratamientos teóricos sobre el alma, sino fundamentalmente a las consideraciones prácticas, de tipo fenomenológico, acerca de cómo opera la cura de ciertas dolencias del alma ${ }^{1}$.

Estructuraremos nuestro trabajo en dos partes, las cuales nos permitirán analizar la cuestión desde dos ópticas diferentes. Primero, dilucidaremos el estatuto epistemológico bajo el cual Evagrio comprende la "psicoterapia" que practica. Esto nos permitirá acceder a la imagen normativa de su arte terapéutico tal como él mismo la expone en su obra. En un segundo punto delimitaremos el valor y el aporte que supone para las ciencias contemporáneas de la salud mental, la particular concepción evagriana acerca del estatuto epistemológico de la cura del alma.

\section{HORIZONTE TEOLÓGICO-MORAL DE LA COMPRENSIÓN EVA- GRIANA DE LA SALUD DEL ALMA}

La concepción evagriana de enfermedad se enmarca en un tratamiento fundamentalmente teológico. Evagrio concibe la enfermedad del alma como consecuencia inmediata de la pérdida de la unidad original en la que el nous (luego devenido alma) fue creado. Ahora bien, esta apelación a un horizonte teológico moral no supone en absoluto un distanciamiento respecto a las competencias epistemológicas de su práctica psicoterapéutica.

Para Evagrio el trastorno básico es de carácter espiritual y solo secundariamente-aunque no sin ignorar y ocuparse de su especificidad psíquica-aparecen los síntomas correlativos que afectan al hombre en relación con su cuerpo, con su alma, con sus estructuras emotivas-cognitivas, y sus relaciones con los demás hombres.

El Póntico presenta la caída del nous como condición de posibilidad del enfermar humano. Dicha caída, en efecto, pone al nous en un estado parà phýsin por el cual se desquician todas las partes del alma persiguiendo un fin para el cual no han sido hechas. La parte pasional busca la satisfacción del cuerpo y la

1 Ciertamente nuestro estudio parte del supuesto de que la psicoterapia contemporánea tiene su fuente y origen en antiguas tradiciones filosóficas y religiosas. La idea que dicha práctica deriva de aproximaciones estrictamente científicas ha sido cuestionado por Perrez (1989) y Sollod (1982) entre otros. 
actividad cognitiva se desarrolla partiendo de las premisas dadas por las pasiones, propiciando una labor imaginativa, memorativa y racional que aleja al hombre de la realidad. De este modo, y tal como explica Larchet $(2007,13)$, es la caída, lo que instaura en el alma "un estado multiforme de enfermedad".

En sus descripciones de lo que hoy reconocemos como cuadros nosológicos de las dolencias del alma el monje del Ponto destaca, como vemos, un síntoma axial, a saber, un trastorno de la relación de la persona respecto a su fin. El hombre caído está alejado y desviado de su fin natural. Éste no es sino un nous encarnado que padece un dinamismo cognitivo-pasional -propio de su condición caída- que inclina sus facultades a obrar de modo contrario a su naturaleza, persiguiendo fines que no son aquellos para los cuales fue creado.

Evagrio en reiteradas ocasiones emplea categorías médicas para describir la desviación moral del hombre respecto a su fin propio. Y para ello, a lo largo de toda su obra, utiliza dos expresiones caras al corpus hipocrático y a la con-

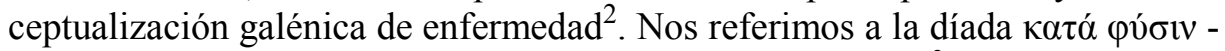
$\pi \alpha \rho \alpha ́$ $v$ $\sigma ı$ (acorde a la naturaleza/contrario a la naturaleza) ${ }^{3}$.

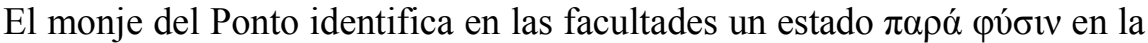
medida que ellas manifiestan un movimiento o un estado contrario a su finalidad propia. Los logismoi, o pensamientos malvados, no son sino estructuras cognitivas que profundizan el movimiento $\pi \alpha \rho \alpha ́$ pv́бıv del alma hacia la nada o hacia las cosas vanas. Es decir, hacia todas aquellas cosas que impiden u obstaculizan la contemplación de la unidad.

En contraposición a este estado de enfermedad, el Evagrio $(1971,56)$ iden-

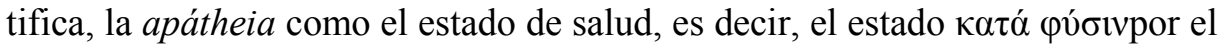

2 Respecto a la presencia de nociones remontables a la medicina hipocrático-galénica en Evagrio, es Dysinger $(2004,2005,104-30)$ quien más ampliamente se ha expedido. Este autor comprueba que el uso del lenguaje médico, muy común en los predecesores de Evagrio y aún en su misma atmósfera intelectual, alcanza una extensión inusitada en el monje del Ponto. Para ampliar el estudio respecto a la vinculación de Evagrio con la medicina antigua se pueden ver los estudios de Refoulé (1961, 506-511); Guillaumont (1971, 545); Messana (1999, 102); Casiday (2013, 13); Tsakiridis $(2010,39)$; Pereto Rivas $(2016,5-6,10-13)$. Respecto a la presencia de las teorías médicas antiguas en la época y en la atmósfera intelectual de Evagrio puede consultarse: Alby (2015); BoudonMillot \& Pouderon (2005).

3 Galeno (2006, 180-301) tiene una definición de enfermedad confluyente con esto: "enfermedad es dicho de cualquier constitución contraria a la naturaleza, por la cual la función está primariamente dañada" (Sobre las diferencias de los sintomas (De symptomatum differentiis, VII.43K). En Sobre las diferencias de las enfermedades (De differentiis morborum, II.1 VI.836-8K, se puede encontrar una definición confluyente (Galeno 2006, 131-156).

Por lo demás, como lo ha demostrado Laín Entralgo (1970, 55-194) con abundancia de citas, la utilización de la diada katà phýsin/parà phýsin es fundamental en el corpus hipocrático, y la enfermedad en el marco de éste se entiende como desorden de la phýsis y, en este sentido, como estado parà phýsin. 
cual las facultades del alma están orientadas habitualmente al placer espiritual

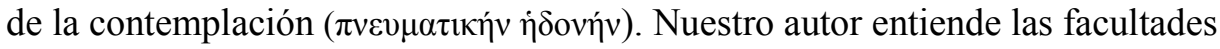
como auxilios que le han sido dados al hombre para re-acceder a la ciencia de la Trinidad Santa.

En suma, lo que define el funcionamiento sano o enfermo de una facultad es el hecho de que su dinámica se oriente o no al fin propio del hombre que es el conocimiento de la unidad.

Las obras evagrianas en las que hoy parecen reconocerse, aparentemente, valiosas proyecciones psicoterapéuticas se desenvuelven en este contexto teológico. El estado protológico y escatológico de unidad, la caída del nous, el estado postlapsario y el itinerario de retorno a la unidad, no son sino el horizonte semántico que da cuenta de su particular comprensión de la enfermedad del alma. El autor ordena las prácticas de valor psicoterapéutico a un fin específico que no es otro sino el de alcanzar la beatitud del alma.

Ahora bien, cabe aquí retomar nuestra objeción inicial. ¿Si el tratamiento que hace Evagrio de ciertas prácticas se enmarca en una comprensión teológica de la salud del alma, es lícito hablar, hoy en día, del valor psicoterapéutico de sus prácticas y doctrina? ¿Acaso puede predicarse valor psicoterapéutico a prácticas que poseen una intencionalidad específicamente religiosa? El reiterado uso de figuras médicas por parte de Evagrio, para explicar estados, dinámicas y procesos del alma ¿no debería interpretarse como un mero recurso retórico, como una extensión analógica de la salud física del cuerpo a la salud espiritual del alma?

Ciertamente, tal como acabamos de apuntar, nuestro autor reconoce en el alma enferma una etiología eminentemente teológica. Ahora bien, ello no es óbice en absoluto para que el monje del Ponto desarrolle prácticas psicoterapéuticas que atiendan a dinámicas propiamente psíquicas. En su doctrina de los ocho logismoi propone una serie de prácticas en orden a curar los procesos de desequilibrio pasional y de distorsión cognitiva a los que dicho estado de enfermedad espiritual da lugar. En este sentido, no puede decirse que su cura del alma se limite a corregir o revisar las determinaciones fundamentales del enfermo. $\mathrm{Su}$ experiencia monacal de autoconocimiento y de padre espiritual de otras almas parece mostrarle que el camino ético de cura del alma que propone debe complementarse y a veces partir de la utilización de otros recursos de índole estrictamente psíquica. Por ejemplo, en el camino de liberación de los logismoi que la afectan.

Evagrio entiende originalmente a estos pensamientos malvados no como pecados capitales sino como estructuras de pensamiento que se dan 
necesariamente a lo largo de la evolución psíquica-espiritual de la persona. En este sentido éstos se acercarían más al modelo de los pensamientos obsesivos que a las estructuras de pecado ${ }^{4}$. Ahora, para poder liberar al alma de dichos pensamientos no se limita a apelar a la voluntad del alma que está bajo su cuidado sino a corregir -mediante las imágenes a las que se alude en la salmodialas dinámicas cognitivas y emotivas que se muestran afectadas por un logismos particular.

El nudo gordiano de sus procedimientos curativos se centra en corregir, remover, o neutralizar las estructuras emotivas y cognitivas que operan como causas próximas de las dolencias del alma. La palabra del gnóstico, la recitación rítmica de la salmodia, la confutación y el desenmascaramiento son prácticas que se ordenan no sólo modificar simplemente estructuras teóricas, ni mover a voluntad, sino a resignificar mediante nuevas experiencias la valoración que, tamizadas por un logismoi o por una inclinación pasional desordenada, el discípulo tiene respecto de sí mismo, de las circunstancias y/o de los demás.

Este particular tratamiento que recomienda al alma enferma nos da una pista respecto a su comprensión de la psicoterapia que practica. Su cura del alma parece ocuparse fundamentalmente del enfermar psíquico del alma, constituido por esa zona, por decirlo de algún modo, en la que el alma actúa y opera en cuanto está unida al cuerpo. Ciertamente que esta zona no está cerrada en sí misma sino ordenada y abierta al espíritu. Entre ella y éste se da una admirable economía de mutuo influjo.

Evagrio integra o subsume en sus prácticas psicoterapéuticas el tratamiento galénico acerca de los estados morbosos del alma.

Por un lado, sigue la tesis que Galeno (2003) desarrolla en Quod Animi Mores Corporis temperamenta Sequntur ${ }^{5}$, a saber, que las disfunciones humorales son causa per accidens de las afecciones anímicas. El médico de Pérgamo postula que, a causa de una alteración humoral, el alma puede verse accidentalmente impedida para ejercer sus funciones o facultades: sea la memoria, la inteligencia o el mismo movimiento voluntario, dando lugar a diversas enfermedades mentales. En línea con esta tesis Evagrio argumenta que muchas acciones o movimientos del alma derivan de la krásis del cuerpo, es decir, se trata de perturbaciones psíquicas que tienen un origen físico. De aquí que la acción del

4 Ciertamente estos logismoi pueden devenir en pecado. Este puede dar lugar a una especie de estratificación de los desórdenes pasionales que va de los episodios excepcionales de pasión exacerbada a hábitos inveterados de conducta. Pero Evagrio se preocupa principalmente por entender al logismos como una estructura cognitiva que se resuelve tanto en una dimensión psíquica como sobrenatural.

5 "El temperamento del cuerpo transforma las funciones del alma", dice Galeno $(2003,779)$ en una fórmula que sintetiza la idea de toda esta obra. 
demonio se oriente muchas veces, a desequilibrar o alterar las krásis $^{6}$ humorales del cuerpo, a fin de provocar en el alma fantasías, pensamientos o reflexiones que la alejen de su fin último que es el conocimiento ${ }^{7}$.

Por otro lado, Evagrio también reconoce la otra cadena de causalidad que Galeno alude para explicar las afecciones morbosas del alma, a saber, que las pasiones o costumbres de alma provocan alteraciones fisiológicas en el órgano que tienen como sede $y$, a partir de ese movimiento, se comportan como causa de una disfunción humoral que afecta a la salud del cuerpo (Galeno 2016, 85). En Diagnóstico y tratamiento de las pasiones del alma de cada uno; y Diagnóstico y tratamiento de los errores del alma de cada uno, Galeno (2013) extiende la tarea médica a reconocer no solo la disfunción humoral del paciente sino también las pasiones y los errores acerca del fin de la vida que pueden estar provocando la disfunción orgánica. En este sentido, escribe, "El médico debe considerar como parte de su tarea, no sólo descubrir la idiosincrasia del paciente, sino también averiguar qué es lo que oprime su mente" (Galeno, 2013, 184). Pues tanto las pasiones como los hamartemata ${ }^{8}$, los juicios errados acerca del fin de la vida ${ }^{9}$, pueden comportarse como la condición fundante de su enfermar (Galeno, 2015).

Esta práctica de la medicina que integra en su mismo quehacer el diagnóstico acerca de las costumbres del alma -es decir, tanto sus hábitos cognitivos como comportamentales-, es el que incorpora y desarrolla Evagrio en sus

6 Evagrio en los capítulos sesenta y tres y ochenta y tres del tratado Sobre la Oración parece estar usando aquí el término akrasía no en el sentido de ausencia de mezcla como haría suponer la alfa privativa, sino como "mala mezcla". Quizá el término griego preciso sería el de $\delta v \sigma \kappa \rho \alpha \sigma i ́ \alpha$ (Evagrio, 1999). Este término al igual que en la obra evagriana, tampoco es usado, nos anoticia Laín Entralgo (1970, 193), en el Corpus Hipocrático, "pero sí, y con todo rigor, la idea por él expresada".

7 Cabe destacar aquí que Evagrio no reduce el modelo explicativo de un trastorno a una intervención demoníaca. Ciertamente afirmaban que esta intervención era posible en cualquier enfermedad, más no por ello dejaba de estudiar las disposiciones psíquicas y espirituales que las facilitaban, o que resultaban de aquellas.

8 Galeno en numerosos pasajes de su De Animi Cuiuslibet Peccatorum Dignotione et Curatione (1997) utiliza esté término 5.85.5; 5.59.5; 5.59.11; 60.5; 60.10; 61.5; 63.9; 63.15; 64.3; $76.12 ; 77.2 ; 77.5 ; 77.11 .12 ; 77.14 ; 78.4 ; 78.11 ; 91.7 ; 97.2 ; 103.15$.

9 También se refiere a ellos con el término $\pi \alpha \rho o ́ \rho-\bar{\alpha} \mu \alpha, \alpha \tau o \zeta$, que se puede traducir como equivocación, yerro, cosa mal vista. Esta palabra está a su vez emparentada con el verbo $\pi \alpha \rho o ́ \rho \alpha \sigma ı \zeta$,

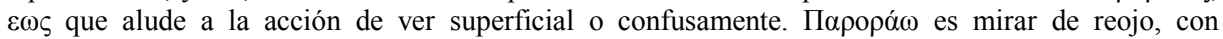
indiferencia o descuido.

En Pecc. Dig. 5.64.3- 5.72: escribe lo siguiente: “Ahora, es posible comprender claramente en qué se diferencia la pasión del error. Imaginemos que un hombre haya elegido como doctrina hacer el bien a los hombres, sobre la base de que ese era su verdadero fin. Pero por causa del sueño, la pereza, la búsqueda del placer o algún motivo semejante, renuncia a ayudar: en este caso es engañado por una pasión. Por otro lado, si uno ha juzgado bueno procurarse solamente placer o serenidad y por esa razón se abstiene de ayudar a sus conciudadanos o a miembros de la familia cuando son víctimas de una injusticia, esa falla se debe a una opinión desafortunada, no a una pasión". 
tratamientos respecto a la cura del alma. Sus prácticas psicoterapéuticas se mueven naturalmente en una dimensión ética, aunque atendiendo a su vez a las relaciones complejas que tal dimensión presenta con el cuerpo y con las estructuras cognitivas y emotivas sensibles.

Sus prácticas de cura del alma en la medida que incorporan y profundizan la concepción galénica del enfermar parecen encontrar en la medicina su especificidad epistemológica. El cuidado en la medicina galénica se dice no solo del cuerpo sino también del alma y del espíritu. Se trata de una práctica médica que integra la dimensión ética como posible factor etiológico de enfermedad humano. En este sentido, la medicina parece ser el estatus epistemológico en el que se inscribe y se entronca su tarea de cuidar y curar a los otros.

La doctrina de los ocho logismoi del Monje del Ponto podría entenderse como un desarrollo y a su vez, como una delimitación de la tradición galénica, a la cura de las enfermedades propiamente psíquicas. En ella Evagrio estudia particularmente aquellos trastornos donde no existe originariamente una disfunción, una mala krasis, o una lesión orgánica. Se trata más bien de dolencias o problemas emocionales que tienen como factor patógeno el apego desmedido de las facultades a fines problemáticos. Se trata de problemas emocionales o psicológicos que tienen ciertamente una etiología de carácter moral que en Evagrio podría individuada con la noción de filautía.

El monje del Ponto explica que las perturbaciones mentales - pensamientos no deseados y malsanos o "mortales"- no son sino diferentes tipos de pensamientos problemáticos característicos de etapas particulares del desarrollo espiritual. Desde su punto de vista, la persona se ve irremediablemente afectada por tales pensamientos. Aún más, los entiende como etapas evolutivas necesarias y normales en desarrollo espiritual de la persona.

Podríamos reconocer en este tratamiento una particular teoría psicopatológica del desarrollo, la cual reconoce como factor etiológico de los desórdenes anímicos a los apegos que se desarrollan a través de las pasiones. Evagrio alega que, al principio del desarrollo, el alma se enfrenta a todos aquellos pensamientos que tienen que ver directamente con un desorden del apetito concupiscible: gula, lujuria y avaricia. En la medida que la persona progresa espiritualmente tiene que habérselas con la tristeza y la ira, pensamientos propios de la facultad irascible. (Sinkewicz 2003, 214-215; Dysinger 2005, 21; Cassiano 1999, 69-193). Finalmente, se siguen aquellos pensamientos propios de la facultad racional. Se trata de aquellas ideas irracionales sobre nuestros logros y nosotros mismos que tienden a estar afectadas por el deseo de vanagloria o de elogio de los demás, falta de humildad 
u orgullo. En esta visión de las cosas, el sano desarrollo de la personalidad se traduciría en la apatheia o desapego de los deseos desordenados que se manifiesta en la estabilidad emocional, el control del comportamiento y la ausencia de distracciones en la oración (Driscoll 2005, 22-24).

La extensión de la comprensión médica a las dolencias del alma exige a Evagrio la tarea de discriminar los procesos de desequilibrio pasional y de distorsión cognitiva concomitantes a una particular dinámica espiritual. En este sentido, cabe destacar su esfuerzo intelectual no solo por interpretar teológicamente una vivencia espiritual sino también por dar cuenta de las dinámicas psicológicas que subyacen a ella. Desde un particular marco teológico, desarrolla una semiología que permite discernir y sistematizar los signos y la evolución propia de un estado espiritual. Analiza fenomenológicamente las dinámicas valorativas que subyacen a los diversos procesos o conflictos espirituales.

Ciertamente la comprensión evagriana del enfermar humano se resuelve en último término en una compresión teológica del hombre. La caída original es en definitiva el origen del desorden pasional e incluso humoral en el que se expresa el estado de enfermedad. Mas la terapia o la cura del alma para nuestro autor no puede escindirse de las dinámicas psico-fisiológicas e incluso éticas que la afectan.

De este modo podríamos decir que la sede epistémica de muchas de las prácticas de la cura del alma es, en nuestro autor, fundamentalmente médicas. Aunque esta tarea médica no puede entenderse en un sentido unívoco. Así como Galeno entendió la necesidad de ocuparse de aquellas dinámicas éticas y cognitivas que afectan al enfermo, Evagrio apela también a estructuras psíquicas y morales. Y esto no supone en absoluto un distanciamiento respecto a la intencionalidad médica de sanar el alma. Por el contrario, implica un cumplimiento aún más acabado pues se estaría ocupando de aquella dimensión específicamente humana que también se comporta como agente causal de su enfermar.

En esta misma línea es necesario destacar que el objetivo de la doctrina evagriana de los logismoi no es presentar los pensamientos como condicionantes del actuar moral sino causas etiológicas primarias del enfermar anímico. Esta clave hermenéutica es la que nos permite dar cuenta de la continuidad que existe entre sus consejos de tono moral y sus observaciones de carácter "clínico". Dicho en otros términos, si la dimensión moral y espiritual del alma es parte de los "intereses clínicos" de Evagrio es porque entiende -en línea con la doctrina 
galénica de las enfermedades del alma- que ella afecta directamente a la salud espiritual del hombre.

La salud humana, para nuestro autor no puede ser explicada de modo autoconsistente, ni por la medicina ni por la moral. Cada campo no es sino un semicírculo que deben recorrerse recíprocamente. La complementariedad explicativa de estas dos dimensiones, integrada y subordinada a una síntesis teológica mayor, conforma el círculo explicativo completo. Evagrio no deja de atender a la dependencia del hombre respecto de la naturaleza, pero, sobre todo, destaca la fuerza del espíritu sobre la naturaleza. Desde este horizonte epistemológico el uso de figuras médicas en el tratamiento de cuestiones morales se revela no como un mero recurso retórico sino como expresión de su comprensión epistémica de la cura del alma.

Los escritos de Evagrio se inscriben en un contexto de razonamientos teológicos y filosóficos ordenados a otros fines que no son propiamente los de dilucidar puntualmente el estatuto epistemológico de sus prácticas terapéuticas. No obstante, sus mismas prácticas traslucen una particular comprensión acerca del objeto y del modo de llevar adelante una "praxis psicoterapéutica". Se trata de una práctica que procura dar cuenta de la íntima retroalimentación que existe entre los hábitos morales y las estructuras cognitivas y emotivas patógenas. El nudo gordiano de su comprensión acerca de esta relación entre las afecciones psíquicas y morales se resuelve en sus técnicas psicoterapéuticas.

\section{PROYECCIONES DE LA DOCTRINA EVAGRIANA EN LAS CIENCIAS CONTEMPORÁNEAS DE SALUD MENTAL}

Una vez que hemos explicitado la imagen normativa que tiene el Monje del Ponto acerca del arte terapéutico que practica, es posible dilucidar su potencial y sus posibles proyecciones para la práctica de las escuelas contemporáneas de psicoterapia.

Ciertamente los conocimientos de Evagrio sobre la vida psíquica, obtenidos por la experiencia del monacato, pueden presentar múltiples ventajas en muchos campos de la psicoterapia. Pero aquí puntualmente nos interesa presentar cómo el sustrato eidético acerca de la cura del alma que practica, puede significar un aporte para la discusión actual acerca del estatuto epistemológico de la psicoterapia.

En nuestros días no se encuentran, en absoluto, trazados los límites epistemológicos de la psicoterapia. Por el contrario, ella se nos presenta como 
una práctica de fronteras borrosas. Se trata de un concepto operativo que arrastra desde sus orígenes con una referencia un tanto vaga y genérica. Su ambigüedad semántica, en parte, no es sino el resultado de una larga discusión acerca de si ella debe ser entendida como un instrumento médico o como una práctica ética subsidiaria de la salud espiritual. Mientras que algunas escuelas o tradiciones de psicoterapia se esfuerzan por mostrarse emparentadas con la práctica médica (pensemos los desarrollos de la psiquiatría moderna), otras se definen como una terapia eminentemente moral $-\mathrm{y}$ en este último caso recordemos que en el siglo XIX la psicoterapia se llamaba originalmente terapia moral, justamente en oposición a la terapia corporal ${ }^{10}$-. Esta discusión epistemológica que atraviesa la conformación moderna de las ciencias de la salud mental da cuenta de la existencia del modelo ético y del modelo médico como los dos paradigmas antitéticos fundamentales en los que oscila fundamentalmente la discusión acerca de estatuto epistemológico de la psicoterapia ${ }^{11}$.

Con la intención de radicalizar el planteo podríamos ilustrar el modelo médico con lo que se reconoce hoy en día como la medicina o la psiquiatría basada en la evidencia. Esta tradición que ubica la psicoterapia en el locus de la medicina concibe los problemas mentales primariamente como una disfunción objetiva por cuyos síntomas es susceptible de ser clasificada en categorías discretas de enfermedades al igual que las disfunciones físicas. Pensemos los esfuerzos que caracterizan a la psiquiatría desde la segunda mitad del siglo XVIII por diferenciar y clasificar progresivamente las enfermedades mentales. Existe a la sazón un intento primario de encontrar una base anatomo-patológica de dichas enfermedades, es decir, una lesión cerebral específica en la que sustentar esta clasificación. Luego, junto al fracaso de esta orientación, por no encontrarse ningún tipo de lesión en la mayoría de las enfermedades psíquicas, la posterior adopción del criterio nosotáxico según el cual los trastornos son discernibles en virtud de su etiología, su clínica, su evolución, su pronóstico y su respuesta a un particular tratamiento. En nuestros días podríamos decir que las diversas ediciones del Manual Diagnóstico y Estadístico de los Trastornos

10 Ciertamente que cuando se hablaba de terapia moral se tomaba en sentido amplio, como sinónimo de lo que hoy se llama psíquico o conductual.

11 La medicina moderna tiende a distinguir entre la salud física y espiritual dependiendo si los síntomas de una enfermedad son primariamente físicos o mentales. Naturalmente este dualismo es absolutamente ajeno a la comprensión que el primer monacato cristiano tiene acerca del hombre y la salud. Los padres del desierto conciben la salud como un fenómeno holístico e inclusivo que se refiere a la persona en su integridad: cuerpo, alma y espíritu.

Ahora bien, en cuanto que nuestro objetivo primario es delimitar el alcance psicoterapéutico del tratamiento que hace los padres del desierto nos limitaremos a discernir los dos modelos de salud mental vigentes en la psicología contemporánea. No obstante, es necesario hacer la salvedad de que se trata de un recurso de simplificación que no corresponde con la visión holística de las fuentes. 
Mentales junto a los desarrollos de las neurociencias forman parte medular de los contenidos y estructuras indispensables para pensar la tarea de la psicoterapia desde este modelo médico.

En contraposición a este modelo médico de salud mental, se ha formulado en las últimas décadas lo que se reconoce como el modelo positivo de salud mental. Se trata de una nueva aproximación al concepto de salud humana que presenta las virtudes como una especie de antítesis de los trastornos psicopatológicas categorizados en el DSM. En lugar de entender la salud mental como la ausencia de enfermedad tal como lo hacen las clasificaciones psicopatológicas del DSM, la entiende como el desenvolvimiento positivo de las potencialidades humanas a través de la actividad virtuosa. (Seligman and Csikszentmihalyi 2000; Snyder and McCullough 2000; Seligman 2002; Emmons 2004). La salud humana no es sino el estado de plenitud emocional y espiritual que resulta de la práctica virtuosa. Desde este marco comprehensivo el estatuto epistemológico de la psicoterapia se inscribiría fundamentalmente en una dimensión ética y pedagógica. Ella es concebida, no como parte de la medicina, sino más bien, como una poiesis orientada a la de formación moral del paciente (McCullough, Kilpatrick, Emmons, y Larson 2001; Davidson 2005; Seligman, Steen, Park and Peterson 2005; Peterson and Seligman 2004; Dahlsgaard, Peterson and Seligman 2005; Peterson 2006).

Un antecedente importante de estos planteos los hallamos en la obra del psiquiatra vienes Rudolf Allers (1963, 15-16) desarrollada en la primera mitad del siglo XX, quien formula una psicología antropológica en oposición al modelo médico. Argumenta fundamentalmente que en cuanto que la psicoterapia procura modificar desordenes anímicos o conductuales apelando a las facultades espirituales o mentales del sujeto se aparta del campo propio del arte médico. Dicho en otros términos, la psicoterapia al entrar en relación directa con la libertad del paciente, es una actividad de orden moral que implica necesariamente la luz práctica de la ciencia moral. Ciertamente la inteligibilidad del valor moral -agrega- dependerá del ideario antropológico de la tradición psicoterapéutica y del paciente, mas ello no es óbice para asignarle y reconocerle a la tarea psicoterapéutica un estatuto ético.

Sirvan los modelos citados para ilustrar una compleja y extensa discusión que se extiende hasta nuestros días acerca del estatuto epistemológico de la psicoterapia.

Sea que los desarrollos de Evagrio, se entiendan como un aporte al modelo médico de salud mental, o en el marco del cuidado moral del alma, debe entenderse la continuidad que existe para él entre estos dos abordajes que las 
ciencias contemporáneas de la salud mental han divorciado. A nuestro entender la comprensión galénica acerca del enfermar humano que es asumida y profundizada por Evagrio, tal como mostramos más arriba, es la que es capaz de dar cuenta de la continuidad que los modelos contemporáneos de salud mental tienden a escindir.

El valor fundamental de la obra evagriana es poner de relieve en el examen etiológico de las enfermedades del alma la incidencia que tienen las dinámicas psíquicas en la vida espiritual del hombre y viceversa. La reverberación de lo pasional sensitivo en nuestro espíritu y, a su vez, de los desórdenes espirituales en las esferas sensitivas de la personalidad, se presentan como el objeto propio de su cuidado del alma. Este modelo explicativo supera o trasciende la aproximación pendular que se abre en nuestros días entre el modelo médico y el modelo ético.

Sin duda no ofreceríamos un panorama realista sino se explicitaran los esfuerzos internos tanto del modelo médico como del modelo ético por formular modalidades de psicoterapia cada vez más holística e integrales. Cabe citar, en esta línea, la categoría de "Problema Religioso o Espiritual" (Código V62.89) que fue incluida por psiquiatras transpersonales en la cuarta edición Manual Diagnóstico y Estadístico de los Trastornos Mentales. Esta nueva categoría buscaría contemplar y atender a las dificultades o malestar psíquico que puede suponer la pérdida o el cuestionamiento de la fe, los problemas asociados con la conversión a una nueva fe, o el cuestionamiento de los valores espirituales Z71.7 (V62.89). (American Psychiatric Association 2000, 741).

Esta incorporación puede ser representativa del creciente interés que la psicoterapia contemporánea presta a dinámicas espirituales en tanto partes constitutivas de la personalidad. No obstante, el reduccionismo metodológico que caracteriza a la psicopatología y, por ende a la psicoterapia, no se trasciende simplemente sumando partes. En este caso, la unidad entre el modelo médico y lo moral no se concreta simplemente agregando una nueva categoría en la última edición del DSM. Nunca se ha hablado tanto en el seno de la psiquiatría y de la psicología como de la necesidad de acceder a una imagen holística de la práctica de la psicoterapia. Ahora bien, esta imagen holística no se cura con modelos inter-disciplinarios; el enfermar del hombre no es una cuestión de derivación de principios, leyes y constantes subyacentes comunes que pueden explicitar disciplinas tales como la sociología, la bioquímica, la neurociencia, o la psicofarmacología, o la misma teología.

La tarea psicoterapéutica exige abordar el hecho psicopatológico como una unidad que supone la interrelación e interconexión común de todas sus 
dimensiones (Peacocke 1993, 42; Barbour 1997, 230). Ciertamente los diferentes niveles de organización -sean neurológicos, sociales, éticos, psíquicos o religiosos- tienen propiedades únicas que deben ser estudiados respectivamente por una disciplina específica. Pero la práctica de la psicoterapia exige fundamentalmente comprender las conexiones funcionales de todas estas variables en el enfermar humano. Y, en este sentido, creemos que aquí radica el valor de los escritos de Evagrio, representativos por lo demás de toda una comprensión integral del hombre característica de la Antigüedad y la Tardoantigüedad. El monje del Ponto concibe y aborda la unidad funcional que existe entre estas dinámicas, aunque naturalmente en el marco de los incipientes desarrollos de la medicina de su época.

\section{CONCLUSIÓN}

Naturalmente, frente al proyecto de la psiquiatría y la psicología modernas de alcanzar clasificaciones noseológicas claras, los desarrollos de Evagrio podrían ser juzgados como un verdadero retroceso. Las clasificaciones psicopatológicas de los diversos tipos de depresión alcanzadas en el DSM -por citar solo un caso ilustrativo- parecerían ser mucho más exactas, pormenorizadas, medibles y precisas que el vago y genérico concepto de tristeza o de acedia, de los desarrollos evagrianos. Ciertamente podría establecerse una clara correspondencia entre dicha noción y la sintomatología de las psicosis maniacodepresivas o trastorno bipolar de nuestros actuales manuales de psicopatología. Pero no son estas meras correspondencias o continuidades sintomatológicas las que pueden presentar un relativo interés para las ciencias contemporáneas de la salud mental.

A nuestro entender es la imagen normativa, es decir, su particular comprensión de la psicoterapia que practica la que representa verdaderas y auténticas proyecciones para la compleja discusión, de nuestros días, acerca del estatuto epistemológico de la psicoterapia.

Tal como mostramos en el primer punto, Evagrio formula prácticas orientadas a la cura y el cuidado del alma enferma. Se trata de una terapia que, en continuidad con la tradición de la medicina galénica, se ocupa de la íntima retroalimentación que existe entre los hábitos morales o costumbres del ama y las estructuras cognitivas y emotivas patógenas. Esto nos permite concluir no solo acerca de la pertenencia y la legitimidad de predicar un potencial terapéutico a su doctrina. sino también destacar su aporte a uno de los problemas 
medulares de las ciencias de la salud mental como es el del ordenamiento epistemológico de la psicoterapia.

\section{REFERENCIAS BIBLIOGRÁFICAS}

Alby, Juan Carlos. 2015. La medicina filosófica del Cristianismo antiguo. Santa Fe: Universidad Católica de Santa Fe.

Allers, Rudolf. 1963. Existencialismo y psiquiatría. Dobrynski E. A. (trad.). Buenos Aires: Troquel.

Barbour, Ian. 1997. Religion and science: Historical and contemporary issues. San Francisco CA: Harper San Francisco.

Boudon-Millot, Veronique, and Pouderon, Bernard. 2005. Les Pères de l'Eglise face à la science médicale de leur temps. Actes du IIIe Colloque international d'études patristiques. Paris: Beauchesne.

Bradford, David. 2011. "Brain and Psyche in Early Christian Asceticism", Psychological Reports 109 (2): 461-520. Accessed February 28, 2012. doi: 10.2466/07.09.16.20.PR0.109.5.461-520.

Bradford, David. 2012. "Evagrius Ponticus and the Psychology of 'Natural Contemplation"”, Studies in Spirituality 22, 109-125.

Casiday, Augustine. 2013. Reconstructing the theology of Evagrius Ponticus: Beyond heresy. Cambridge: Cambridge University Press.

Corrigan, Kevin. 2017. Trauma before Trauma: Recognizing, Healing and Transforming the Wounds of Soul-Mind in the Works of Evagrius of Pontus, by Ilaria Ramelli, Kevin Corrigan, Giulio Maspero and Monica Tobon (eds.), Studia Patristica LXXXIV: Evagrius between Origen, the Cappadocians and Neoplatonism, Leuven: Peeters.

Dahlsgaard, Katherine, Peterson Christopher, and Seligman, Martin. 2005. "Shared virtue: The convergence of valued human strengths across culture and history". Review of General Psychology, 9: 203-213. Accessed February 28, 2012. doi: 10.1037/1089-2680.9.3.203

Davidson, Richard. 2005. "Well-being and affective style: Neural substrates and biobehavioural correlates". In F. Huppert, N. Baylis, \& B. Keverne (eds.), The science of well-being (pp. 107-139). Oxford: Oxford University Press.

Driscoll, Jeremy. 2005. Steps to spiritual perfection: Studies on spiritual progress in Evagrius Ponticus. New York: Newman Press.

Dysinger, Luke. 2005. Psalmody and prayer in the writings of Evagrius Ponticus. Oxford: Oxford University Press.

Evagrio Póntico. 1971. Traité pratique ou le moine. Edited and translated by Antoine Guillaumont and Claire Guillaumont. Tomo II. Paris: Cerf. 
Evagrio Póntico. 1999. La preghiera. Notes and translated by Vicenzo Messana. Roma: Città Nuova.

Fernández Samuel. 1999. Cristo médico, según Orígenes: La actividad médica como metáfora de la acción divina. Roma: Augustinianum, 17-53.

Galeno. 1997. De propium Animi Cuiuslibet Affectuum Dignotione et Curatione. Galen: Selected Works. Notes, introduction and translated by P. N. Singer. Oxford -New York: Oxford University Press.

Galeno. 2003. Las facultades del alma siguen los temperamentos del cuerpo. Notes, introduction and translated by Juana Zaragoza Gras. Madrid: Gredos. Galeno. 2006. On Diseases and Symptons. Notes, introduction and translated by Ian Johnston, New York: Cambridge University Press: 180-301.

Galeno. 2013. De las pasiones y los errores del alma. Galeno de Pérgamo Traducción y prólogo por Liliana Cecilia Molina González. Colombia: Editorial Universidad de Antioquía.

Galeno. 2015. On the doctrines of Hippocrates and Plato. Edition, translation and commentary by Phillip de Lacy. Vol III. Thrid edition. Berlin: AkademieVerlag.

Galeno. 2016. Sobre la conservación de la salud. Introducción, traducción, notas e Îndices de Inmaculada Rodríguez Moreno. Madrid: Ediciones Clásicas.

García Ballester, Luis. 1996. "Alma y cuerpo, enfermedad del alma y enfermedad del cuerpo en el pensamiento médico de Galeno". Revista de la Asociación Española de Neuropsiquiatría XVI/60: 705-735.

Gianfrancesco Angelo. 2008. "Monachisme ancien et psychopathologie". L'evolution psychiatrique 73 (1): 105-26.

Guillaumont, Antoine, and Guillaumont, Claire. 1971. Introducción y comentarios, en Evagrio Póntico, Traité pratique, ou, Le moine SC 170-171, Paris: Éditions du Cerf.

Griver Inbar. 2016. "The Paradoxical Effects of Attentiveness". The Journal of Early Christian Studies 24 (2): 199-227.

Graiver Inbar. 2018. "Possible Selves in Late Antiquity: Ideal Selfhood and Embodied Selves in Evagrian Anthropology". The Journal of Religion 98 (1): $59-89$.

Hill Jonathan. 2010. "Did Evagrius Ponticus (AD 346-99) have obsessivecompulsive disorder?" Journal of Medical Biography 18(1): 49-56. Accessed April 28, 2012. doi: 10.1258/jmb.2009.009056.

Lain Entralgo, Pedro. 1970. La Medicina Hipocrática. Madrid: Ediciones de la Revista de Occidente.

Lain Entralgo, Pedro. 2005. La curación por la palabra en la Antigüedad Clásica. Barcelona: Anthropos.

Larchet Jean Claude. 2001. Théologie de la maladie. Paris: Cerf. 
Larchet Jean Claude. 2003. Terapia delle malattie spirituali, un'introduzione alla tradizione ascetica della Chiesa Ortodossa, Milano: San Paolo.

Larchet Jean Claude. 2007. Thérapeutique des maladies spirituelles. Paris: Cerf. Larchet Jean Claude. 2006. ID, L'inconscio spirituale, malattie psichiche e malattie spirituali, Milano: San Paolo.

Messana, Vicenzo. 1999. "Introduzione”. En Evagrio Póntico, La preghiera, edited by Vicenzo Messana, 5-54. Roma: Città Nuova.

McCullough, Michael, Kilpatrick, Shelley, Emmons, Robert. and Larson, David. 2001. "Is gratitude a moral affect?" Psychological Bulletin 127 (2): 249-266. Accessed April 28, 2012. doi: 10.1037/0033-2909.127.2.249.

Seligman, Martín. 2002. "Positive psychology, positive prevention, and positive therapy". In C. R. Snyder, \& S. J. Lopez (Eds.), Handbook of positive psychology (pp. 3-9). New York: Oxford University Press.

Seligman, Martín, and Csikszentmihalyi, Mihaly. 2000. "Positive psychology: An introduction". American Psychologist 55 (1), 5-14. Accessed April 28, 2012. doi: 10.1037//0003-066x.55.1.5.

Seligman, Martín, Steen, Tracy, Park, Nansook \& Peterson, Christopher. 2005. "Positive psychology progress: Empirical validation of interventions". American Psychologist 60 (5), 410-421. Accessed April 28, 2012. doi: 10.1037/0003-066X.60.5.410

Snyder, Charles, and McCullough, Michael. 2000. A positive psychology field of dreams: "If you build it, they will come ...". Journal of Social and Clinical Psychology, 19 (1), 151-160. Accessed April 28, 2012. doi:10.1521/jscp.2000.19.1.151.

Peacocke, Arthur. 1993. Theology for a scientific age: Being and becomingnatural, divine, and human. Minneapolis, MN: Fortress Press.

Peretó Rivas, Rubén. 2016. "El conocimiento de sí como sanación en Evagrio Póntico", en I Jornadas Internacionales de Filosofía "El cuidado de sí y el cuidado del mundo" (24, 25 y 26 de agosto de 2016), Buenos Aires, Universidad del Salvador.

Peretó Rivas Rubén. 2017. "ACT (Acceptance and Commitment Therapy) y Evagrio Póntico. Algunas correspondencias teóricas", Cauriensia 12, 579598. Accessed January 12, 2018. doi:10.17398/1886-4945.12.579

Perrez, Meinard. 1989. "Psychotherapeutic Methods: Between scientific foundation and everyday knowledge", New Ideas in Psychology 7 (2), 133145. Accessed January 12, 2018. doi: 10.1016/0732-118X(89)90019-6.

Peterson Christopher and Seligman, Martín. 2004. Character strengths and virtues: A handbook and classification. Washington, DC: American Psychological Association 
Peterson, Christhpher. 2006. "The Values in Action (VIA) classification of strengths". In Mihaly Czikszentmihaly and Isabella Czikszentmihalyi (Eds.), A life worth living: Contributions to positive psychology (pp. 29-48). New York: Oxford University Press.

Ramelli, Illaria. 2015. "Commentary", en Evagrius's Kephalaia Gnostica: A New Translation of the Unreformed Text from the Syriac, Writings from the Greco-Roman World. Atlanta: SBL Press.

Refoulé, François. 1961. "Rêves et vie spirituelle d'après Évagre le Pontique", Supplément de la Vie Spirituelle 59: 506-511.

Sollod, Robert. 1982. "Non-scientific sources of psychotherapeutic: A case for inclusion". In E. P. Shafranske (ed.) Philosophy, religion and psychotherapy, 41-56. Washington DC: University press of America.

Trader, Alexis. 2012. Ancient Christian Wisdom and Aaron Beck's Cognitive Therapy. A meeting of minds. American University studies, New YorkOxford: Peter Lang.

Tsakiridis George. 2010. Evagrius Ponticus and Cogntive Science. A look at moral evil and the thoughts, Eugene, Oregon: Pickwick Publications.

Vasquez Santiago. 2015. Las implicancias psicopatológicas de la acedia en Evagrio Póntico. Revista Latinoamericana de Psicopatología 18(4): 679-703.

María Teresa Gargiulo Universidad Nacional de Cuyo Centro Universitario M5502JMA Mendoza (Argentina) https://orcid.org/0000-0003-3580-9478

Santiago Hernán Vázquez Universidad Nacional de Cuyo Centro Universitario M5502JMA Mendoza (Argentina) http://orcid.org/0000-0002-2388-7172 
